\title{
Paclitaxel and Trastuzumab as Maintenance Therapy in Patients with HER2-Positive Metastatic Breast Cancer Who Underwent High-Dose Chemotherapy and Au- tologous Hematopoietic Stem Cell Transplantation
}

\author{
Yee Chung Cheng ${ }^{1,{ }^{*}}$, Gabriela Rondón ${ }^{1}$, Paolo Anderlini ${ }^{1}$, Issa F. Khouri ${ }^{1}$, Richard E. Champlin ${ }^{1}$, Naoto T. \\ Ueno ${ }^{1,2} \bowtie$ \\ 1. Departments of Stem Cell Transplantation and Cellular Therapy and \\ 2. Departments of Breast Medical Oncology, The University of Texas MD Anderson Cancer Center, Houston, TX, USA. \\ * Present address: Division of Hematology and Oncology, Department of Medicine, Medical College of Wisconsin, Milwaukee, WI.
}

$\square$ Corresponding author: Naoto T. Ueno, MD, PhD, Breast Cancer Translational Research Laboratory, Departments of Breast Medical Oncology and Stem Cell Transplantation and Cellular Therapy, The University of Texas MD Anderson Cancer Center, 1515 Holcombe Boulevard, Unit 1354, Houston, TX 77030; E-mail: nueno@mdanderson.org

() Ivyspring International Publisher. This is an open-access article distributed under the terms of the Creative Commons License (http://creativecommons.org/ licenses/by-nc-nd/3.0/). Reproduction is permitted for personal, noncommercial use, provided that the article is in whole, unmodified, and properly cited.

Received: 2013.05.28; Accepted: 2013.06.25; Published: 2013.09.27

\begin{abstract}
We examined the feasibility and safety of using paclitaxel and trastuzumab as maintenance therapy after high-dose chemotherapy (HDC) with autologous hematopoietic stem cell transplantation (AHST) for patients with HER2-positive metastatic breast cancer. Ten patients ( 9 women and I man) were enrolled in the study. The median age was 46.5 years (range, 27-65 years). The median follow-up time was 1003 days (range, 216-2526 days). All patients had metastatic disease, but 2 had only bone metastasis. One patient had complete response, 6 had partial response and 3 had stable disease to the standard-dose chemotherapy prior to transplantation. The conditioning regimen consisted of cyclophosphamide, carmustine, and thiotepa. After AHST, patients received weekly paclitaxel for 12 doses and trastuzumab every 3 weeks for I year as maintenance therapy. All patients experienced successful engraftment. The only grade 4 toxic effects observed were leukopenia and thrombocytopenia. The most common grade 3 toxic effect was neutropenic fever. No treatment-related deaths were observed. The median progression-free survival time was $44 \mathrm{I}$ days, and the median overall survival time was 955 days. Two patients died in accidents while their disease remained in remission. Five patients died with disease progression. At the time of this report, 3 patients are alive with stable disease, I of whom has remained free of disease progression for 2526 days since transplantation. Our findings indicate that paclitaxel plus trastuzumab as maintenance therapy after HDC with AHST for patients with HER2-positive metastatic breast cancer not only is feasible and safe but also results in survival outcomes similar to historical results.
\end{abstract}

Key words: paclitaxel, trastuzumab, metastatic breast cancer

\section{INTRODUCTION}

High-dose chemotherapy (HDC) with autologous hematopoietic stem cell transplantation (AHST) in patients with metastatic breast cancer has been studied for decades. Several phase II clinical trials of
HDC with AHST in metastatic breast cancer showed overall response rates of more than $50 \%$, and approximately $30 \%$ of patients in those trials survived progression-free for at least 5 years ${ }^{1-10}$. However, the 
results of subsequent phase III clinical trials have been disappointing. Of the 6 randomized trials published to date, 5 showed improvements in time to progression/progression-free survival but not in overall survival11-16, and the sixth showed no benefit in either time to progression/progression-free survival or overall survival ${ }^{17}$. This pattern-improvements in response rates and time to progression but no effect on overall survival-suggests that the major cause of treatment failure after HDC with AHST remains disease relapse, presumably due to residual breast cancer cells that are resistance to treatment. Therefore, measures to improve the outcome of transplantation by eradicating minimal residual disease are a major area of research.

The HER2/neu oncogene (ERBB2) encodes a $185-\mathrm{kDa}$ protein with tyrosine kinase activity that enhances tumorigenicity and metastasis 18,19 . About $20 \%$ to $25 \%$ of breast cancers overexpress the HER2/neu oncogene, and such overexpression is well established to be associated with tumor aggressiveness and worse survival ${ }^{20-22}$. However, HER2-targeted therapy for breast cancer, such as trastuzumab, has improved outcomes significantly. Multiple phase III clinical trials of combined chemotherapy and trastuzumab as adjuvant therapy in patients with high-risk HER2-positive breast cancer have demonstrated a significant improvement in relapse-free survival and overall survival compared to chemotherapy alone $^{23-29}$.

We previously reported that HER2/neu overexpression is a poor prognostic marker for patients with metastatic breast cancer who underwent HDC with AHST $^{30}$. We also previously showed that paclitaxel given to patients with metastatic breast cancer after HDC with AHST results in prolonged disease-free survival $^{31}$. We therefore hypothesized that the combination of paclitaxel and trastuzumab as maintenance therapy after HDC with AHST eradicates minimal residual disease and improves survival in patients with metastatic breast cancer that overexpresses HER2/neu. In the study reported here, we examined the feasibility and safety of using paclitaxel and trastuzumab as maintenance therapy after HDC with AHST for patients with HER2-positive metastatic breast cancer. We also estimated the associated progression-free and overall survival rates.

\section{MATERIALS AND METHODS}

The protocol was reviewed and approved by the Institutional Review Board of MD Anderson Cancer Center. All patients provided written informed consent to participate. Eligible patients were patients with HER2-overexpressing metastatic breast cancer, aged 18 to 65 years, with an Eastern Cooperative Oncology
Group performance status score of 0 or 1 , no major organ dysfunction or active infection, and disease that had responded to standard-dose systemic chemotherapy. Patients with bone disease were eligible if they had experienced clinical improvement of symptoms after standard-dose chemotherapy and did not demonstrate new lesions on radiographic study after such chemotherapy.

Stem cells were collected by filgrastim or filgrastim-plus-chemotherapy mobilization followed by apheresis. Mobilization with filgrastim alone consisted of filgrastim $6 \mu \mathrm{g} / \mathrm{kg}$ by subcutaneous injection every 12 hours for at least 3 to 4 days and until completion of apheresis. For mobilization with filgrastim plus chemotherapy, patients received cyclophosphamide $1.5 \mathrm{~g} / \mathrm{m}^{2}$ by intravenous (IV) infusion over 2 hours on days 1 to 3 with mesna given at a dose of 500 $\mathrm{mg} / \mathrm{m}^{2}$ by IV infusion 30 minutes before the first dose of cyclophosphamide and then $1.5 \mathrm{~g} / \mathrm{m}^{2} /$ day as a continuous IV infusion over 24 hours for 3 days; etoposide $250 \mathrm{mg} / \mathrm{m}^{2} /$ day by IV infusion over 3 hours on days 1 to 3 ; and cisplatin $40 \mathrm{mg} / \mathrm{m}^{2} /$ day by IV infusion over 2 hours on days 1 to 3 . Beginning on day 4 , patients received filgrastim $5 \mu \mathrm{g} / \mathrm{kg}$ by subcutaneous injection daily until completion of apheresis. Apheresis was performed daily until $5 \times 10^{6}$ CD34-positive cells $/ \mathrm{kg}$ was collected. The minimum acceptable cell dose was $3 \times 10^{6}$ CD34-positive cells $/ \mathrm{kg}$. Patients received a minimum of 1 apheresis procedure and a maximum of 5 procedures. Patients with inadequate numbers of progenitor cells harvested were to undergo bone marrow harvest with infusion of both marrow and peripheral blood progenitor cells.

Patients with adequate progenitor cell harvest and no major toxic effects from filgrastim mobilization underwent HDC with AHST. The conditioning regimen was cyclophosphamide, carmustine, and thiotepa. The details of the regimen were as follows (the day of AHST was considered day 0): cyclophosphamide $2 \mathrm{gm} / \mathrm{m}^{2}$ by IV infusion on days $-7,-6$, and -5 with mesna given at a dose of $500 \mathrm{mg} / \mathrm{m}^{2}$ by IV infusion 2 hours before the first dose of cyclophosphamide and then $2 \mathrm{~g} / \mathrm{m}^{2} /$ day as a continuous infusion over 24 hours for 3 days; carmustine $150 \mathrm{mg} / \mathrm{m}^{2} /$ day by IV infusion over 40 minutes on days $-7,-6$, and -5 ; and thiotepa $240 \mathrm{mg} / \mathrm{m}^{2} /$ day by IV infusion over 4 hours on days $-7,-6$, and -5 . The stem cells were thawed and reinfused on day 0. Following AHST, filgrastim $5 \mu \mathrm{g} / \mathrm{kg} /$ day was administered by IV infusion or subcutaneously until the granulocyte count was greater than $1000 / \mathrm{mm}^{3}$ for 3 consecutive days.

Four weeks after AHST, patients received a loading dose of trastuzumab $(4 \mathrm{mg} / \mathrm{kg}$ by IV infusion over 90 minutes) if all the following criteria were ful- 
filled: no progression of disease since AHST; left ventricular ejection fraction at least $45 \%$ and not reduced more than $20 \%$ from the baseline value (before the loading dose of trastuzumab); no irreversible grade 3 or 4 toxic effects from HDC; absolute neutrophil count at least $1500 / \mathrm{mm}^{3}$; platelet count at least $40,000 / \mathrm{mm}^{3}$; hemoglobin level at least $8 \mathrm{~g} / \mathrm{dL}$; creatinine level not greater than $2.0 \mathrm{mg} / \mathrm{dL}$; and total bilirubin level not greater than $2.0 \mathrm{mg} / \mathrm{dL}$. If the above criteria were not fulfilled, treatment could be postponed until up to 10 weeks after AHST. If the criteria were not fulfilled by 10 weeks after AHST, patients were considered off study.

Trastuzumab was administered at a dose of 2 $\mathrm{mg} / \mathrm{kg}$ by IV infusion over 30 minutes every week up to 1 year or until progression of disease. Paclitaxel 80 $\mathrm{mg} / \mathrm{m}^{2}$ by IV infusion over 1 hour was administered weekly for 12 doses starting 3 weeks after the loading dose of trastuzumab. Patients with estrogen-receptor-positive disease received tamoxifen daily if it was clinically indicated and they had not previously experienced disease progression during tamoxifen therapy. Radiation therapy and bisphosphonates were not allowed while patients were receiving the trastuzumab and paclitaxel.

Disease status and left ventricular ejection fraction were evaluated before the trastuzumab loading dose, before the first dose of paclitaxel, after 6 doses of combined trastuzumab and paclitaxel, after 12 doses of combined trastuzumab and paclitaxel, and then every 3 months up to 2 years after AHST, every 6 months during years 3 and 4 , at the end of year 5 , and then yearly. Treatment-related toxic effects were assessed according to the National Cancer Institute Common Toxicity Criteria, version 2.0. Tumor response was assessed according to the Response Evaluation Criteria in Solid Tumors system. Kaplan-Meier analysis was used to estimate survival rates.

\section{RESULTS}

Ten patients were enrolled in the study between October 1, 1999 and May 28, 2003, 9 women and 1 man. The median age was 46.5 years (range, 27-65 years). All 10 patients had metastatic disease, but 2 had only bone metastasis. All 10 patients received at least 1 regimen of standard-dose chemotherapy for their metastatic disease prior to AHST. One patient had a complete response, 6 had partial responses, and 3 had stable disease as best response to standard-dose chemotherapy before AHST. All patients had adequate progenitor cells of $5 \times 10^{6}$ CD34-positive cells/kg collected; no bone marrow harvesting was needed. After transplantation, all patients experienced successful graft enhancement; there were no cases of graft failure. Nine patients received originally planned maintenance therapy of 12-week paclitaxel, and 6 patients received originally planned maintenance therapy of 1-year trastuzumab. Three patients did not finish the maintenance therapy due to progression of disease and 1 patient died in motor vehicle accident on day 216. The treatment-related toxic effects are listed in Table 1 . The only grade 4 toxic effects observed were leukopenia and thrombocytopenia, which occurred in 4 patients each. The most common grade 3 toxic effect was neutropenic fever, and the most common grade 1 and 2 toxic effects were gastrointestinal effects: nausea, diarrhea, and mucositis. No grade 4 cardiac toxic effects were noted. One patient developed a grade 3 cardiac toxic effect, symptomatic decreased left ventricular ejection fraction, but this resolved completely with intervention. No treatment-related deaths were observed.

Table I: Treatment-related toxic effects

\begin{tabular}{llll}
\hline & \multicolumn{3}{c}{ No. of Patients } \\
\hline Effect & Grade 1-2 & Grade 3 & Grade 4 \\
\hline Blood/bone marrow & 3 & 2 & 4 \\
Leukopenia & 0 & 0 & 4 \\
Thrombocytopenia & & & \\
Cardiac & 3 & 1 & 0 \\
Left ventricular systolic dysfunc- & & & \\
tion & 1 & 0 & 0 \\
Hypotension & & & \\
Constitutional & 1 & 0 & 0 \\
Fatigue & 3 & 0 & 0 \\
Nonneutropenic fever & & & \\
Gastrointestinal & 1 & 0 & 0 \\
Anorexia & 6 & 0 & 0 \\
Diarrhea & 4 & 0 & 0 \\
Mucositis & 7 & 1 & 0 \\
Nausea & 0 & 5 & 0 \\
Infectious: neutropenic fever & 1 & 0 & 0 \\
Musculoskeletal: bone pain & & & \\
Neurologic & & 0 & 0 \\
Headache & 1 & 1 & 0 \\
Neuropathy & 2 & 0 & 0 \\
Respiratory & & 0 & 0 \\
Dyspnea & 1 & & \\
Pleural effusion & & & \\
\hline & & &
\end{tabular}




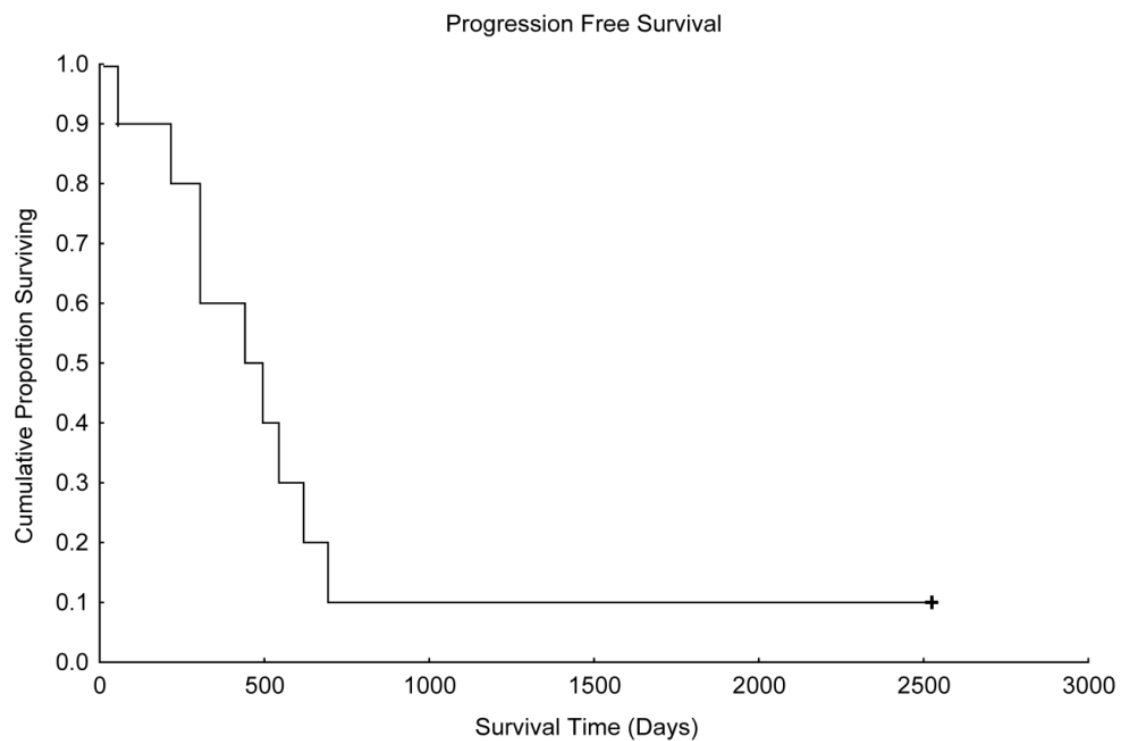

Figure I. Progression-free survival in patients treated with paclitaxel and trastuzumab as maintenance therapy after high-dose chemotherapy and autologous hematopoietic stem cell transplantation for metastatic breast cancer $(\mathrm{N}=10)$.

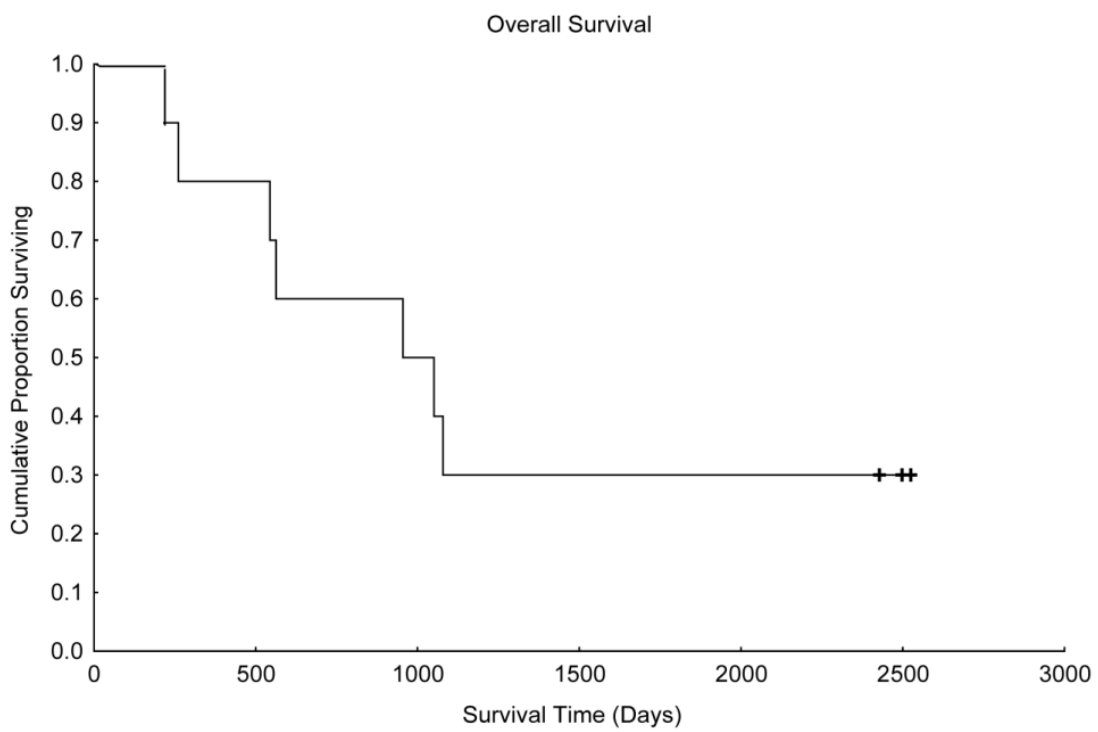

Figure 2. Overall survival in patients treated with paclitaxel and trastuzumab as maintenance therapy after high-dose chemotherapy and autologous hematopoietic stem cell transplantation for metastatic breast cancer $(\mathrm{N}=10)$.

With a median follow up time of 1003 days (range, 216-2526 days), the median progression-free survival time was 441 days (Figure 1), and the median overall survival time was 955 days (Figure 2). Unfortunately, 2 patients died in motor vehicle accidents while their disease remained in remission, 1 on day 216 and 1 on day 544 after AHST. Five patients died with disease progression. At the time of this report, 3 patients are alive with stable disease, including 1 patient who remained continuously free of disease progression 2526 days after AHST. Two other patients had progressive disease diagnosed on day 619 and day 693, respectively, after AHST; in both patients, the disease became stable after salvage treatment. At the time of this report, those patients are alive at 2428 days and 2498 days, respectively, after AHST.

\section{DISCUSSION}

Metastatic breast cancer is considered to be an incurable but treatable disease. With current treatment, the median survival time is about 2 to 3 years. About $5 \%$ of patients live up to 5 years after diagnosis of metastatic disease, and about $2 \%$ live up to 10 years $^{32}$. One of the reasons for this relatively favorable outcome despite the incurable disease is that breast cancer responds to different classes of chemotherapy, including alkylating agents. Therefore, the combination of HDC, particular with alkylating agents, followed by AHST has been studied for decades to improve the outcome of patients with metastatic breast cancer. Unfortunately, treatment failure to HDC with 
AHST still occurs. The failure of metastatic breast cancer to respond completely to HDC with AHST could be due to minimal residual disease owing to intrinsic features of the breast cancer cells that render them resistant to chemotherapy.

One such intrinsic feature is overexpression of the HER2/neu oncogene, which is believed to confer resistance of cancer cells to chemotherapeutic agents ${ }^{33,34}$. Anti-HER2 therapy incorporated into the pre-AHST conditioning regimen or post-AHST maintenance therapy might overcome drug resistance due to HER2 overexpression. In the study reported here, we tested the feasibility and safety of combining paclitaxel with the anti-HER2 agent trastuzumab as maintenance therapy for patients with metastatic breast cancer after HDC with AHST. Results at a median follow-up time of 1003 days (range, 216-2526 days) showed that this combination treatment not only was feasible and safe, associated with no severe cardiac or other toxic effects, but also produced survival outcomes similar to historical results.

Trastuzumab was the first monoclonal antibody developed that specifically targets HER2-positive breast cancer. The major toxic effects of trastuzumab are cardiac toxic effects ${ }^{35,36}$. The incidence of cardiac toxic effects among patients who received trastuzumab alone was $7 \%$, which was similar to the incidence of cardiac toxic effects among patients who received anthracycline-based regimens. However, in patients who received concurrent anthracycline-based chemotherapy and trastuzumab, the incidence of cardiac toxic effects was $28 \%$. Among the patients who had previously received an anthracycline, $11 \%$ of those treated with the combination of paclitaxel and trastuzumab suffered cardiac events, compared to $1 \%$ of those treated with paclitaxel only. The mechanism of trastuzumab-induced cardiac toxic effects and the enhanced toxicity of trastuzumab when the drug is combined with different types of chemotherapy is unknown, but trastuzumab-induced cardiac toxic effects are usually reversible with interruption of treatment. In our study, we did not observe any grade 4 cardiac toxic effects with the combination of paclitaxel and trastuzumab. However, our sample size may have been too small to permit observation of this toxic effect. The only grade 4 toxic effect that we observed was myelosuppression, mostly secondary to the HDC part. The myelosuppression was not permanent, and no life-threatening infection or bleeding was observed.

Currently, several anti-HER2 therapeutic agents besides trastuzumab are approved for HER2-positive metastatic breast cancer. These include lapatinib, pertuzumab, and trastuzumab-emtansine.

Lapatinib is a dual tyrosine kinase inhibitor against HER1 and HER2. It is approved for use in combination with oral capecitabine in patients with metastatic disease that is initially resistant to or recurs after treatment with an anthracycline, a taxane, and trastuzumab. In a phase III trial comparing lapatinib plus capecitabine to capecitabine alone in patients with metastatic breast cancer, the combined treatment showed a benefit in terms of time to progression (median, 6 months versus 4 months) and overall survival (median, 75 weeks versus 65 weeks) ${ }^{37}$. Lapatinib can also be combined with trastuzumab without chemotherapy in patients with metastatic breast cancer. Compared to lapatinib alone, lapatinib plus trastuzumab was associated with improved progression-free survival (median, 11 weeks versus 8 weeks) and overall survival (median, 14 months versus 10 months) $)^{38,39}$.

Pertuzumab prevents dimerization of HER2 with other HER2 family receptors. Compared with trastuzumab and docetaxel in the phase III CLEOPATRA trial, pertuzumab in combination with trastuzumab and docetaxel in patients with metastatic breast cancer was associated with a better response rate $(80 \%$ versus $69 \%)$ and longer progression-free survival (median, 19 months versus 12 months) ${ }^{40}$. In the phase III NeoSphere trial, different combinations of docetaxel, trastuzumab, and pertuzumab were tested as neoadjuvant regimens ${ }^{41}$. The combination of trastuzumab and pertuzumab without docetaxel produced an impressive $17 \%$ pathologic complete response rate. This result was comparable to results achieved with traditional chemotherapy alone but without the side effects usually associated with chemotherapy. These findings suggest that this dual-anti-HER2-therapy approach without chemotherapy might be ideal for long-term maintenance therapy in patients with HER2-positive metastatic breast cancer after HDC with AHST.

Finally, trastuzumab-emtansine is a member of a new class of agents called antibody-drug conjugates. Trastuzumab-emtansine was administered as a single agent since the drug itself is a combination of an anti-HER2 agent and a chemotherapy drug mertansine. In the phase III EMILIA trial, trastuzumab-emtansine was associated with a better response rate ( $44 \%$ versus $31 \%$ ), progression-free survival (median, 10 months versus 6 months), and overall survival (median, 31 months versus 25 months) than standard second-line treatment with lapatinib/capecitabine in patients with metastatic breast cancer ${ }^{42}$. Trastuzumabemtansine consists of trastuzumab bound covalently through the chemical cross-linker SMCC to several molecules of the toxic chemotherapy drug mertansine. The result is a targeted drug that has the efficacy of chemotherapy but does not produce the 
usual chemotherapy side effects because the drug is targeted to HER2-positive cancer cells. This unique antibody-drug conjugate may prove to be more effective than traditional chemotherapy with or without anti-HER2 agents. More research is needed to establish the role of trastuzumab-emtansine in HER2-positive breast cancer.

In summary, our study has shown that for patients with HER2-positive metastatic breast cancer who underwent HDC with AHST, it is feasible and safe to combine an anti-HER2 treatment, trastuzumab, with a cytotoxic chemotherapy agent, paclitaxel, for maintenance therapy after AHST. This combined treatment also produced survival outcomes similar to historical results. However, given the small size of our study, this survival benefit will need to be verified in a larger trial.

\section{ACKNOWLEDGMENTS}

We thank Stephanie Deming for her excellent help in developing this manuscript.

\section{COMPETING INTERESTS}

The authors have declared that no competing interest exists.

\section{REFERENCES}

1. Eder JP, Antman K, Peters W, et al: High-dose combination alkylating agent chemotherapy with autologous bone marrow support for metastatic breast cancer. J Clin Oncol. 1986; 4:1592-7.

2. Peters WP, Shpall EJ, Jones RB, et al: High-dose combination alkylating agents with bone marrow support as initial treatment for metastatic breast cancer. J Clin Oncol. 1988; 6:1368-76.

3. Williams SF, Mick R, Desser R, et al: High-dose consolidation therapy with autologous stem cell rescue in stage IV breast cancer. J Clin Oncol. 1989; 7:1824-30.

4. Williams SF, Gilewski T, Mick R, et al: High-dose consolidation therapy with autologous stem-cell rescue in stage IV breast cancer: follow-up report. J Clin Oncol. 1992; 10:1743-7.

5. Kennedy MJ, Beveridge RA, Rowley SD, et al: High-dose chemotherapy with reinfusion of purged autologous bone marrow following dose-intense induction as initial therapy for metastatic breast cancer. J Natl Cancer Inst. 1991; 83:920-6.

6. Antman K, Ayash L, Elias A, et al: A phase II study of high-dose cyclophosphamide, thiotepa, and carboplatin with autologous marrow support in women with measurable advanced breast cancer responding to standard-dose therapy. J Clin Oncol. 1992; 10:102-10.

7. Antman $\mathrm{KH}$, Rowlings PA, Vaughan WP, et al: High-dose chemotherapy with autologous hematopoietic stem-cell support for breast cancer in North America. J Clin Oncol. 1997; 15:1870-9.

8. Weaver $\mathrm{CH}$, West $\mathrm{WH}$, Schwartzberg LS, et al: Induction, mobilization of peripheral blood stem cells (PBSC), high-dose chemotherapy and PBSC infusion in patients with untreated stage IV breast cancer: outcomes by intent to treat analyses. Bone Marrow Transplant. 1997; 19:661-70.

9. Bensinger WI, Schiffman KS, Holmberg L, et al: High-dose busulfan, melphalan, thiotepa and peripheral blood stem cell infusion for the treatment of metastatic breast cancer. Bone Marrow Transplant. 1997; 19:1183-9.

10. Ljungman P, Bjorkstrand B, Fornander T, et al: High-dose chemotherapy with autologous stem cell support in patients with responding stage IV breast cancer. Bone Marrow Transplant. 1998; 22:445-8.

11. Crump M, Gluck S, Tu D, et al: Randomized trial of high-dose chemotherapy with autologous peripheral-blood stem-cell support compared with standard-dose chemotherapy in women with metastatic breast cancer: NCIC MA.16. J Clin Oncol. 2008; 26:37-43.

12. Roche $\mathrm{H}$, Viens $\mathrm{P}$, Biron $\mathrm{P}$, et al: High-dose chemotherapy for breast cancer: the French PEGASE experience. Cancer Control. 2003; 10:42-7.

13. Biron $P$, Durand $M$, Roche $H$, et al: Pegase 03: a prospective randomized phase III trial of FEC with or without high-dose thiotepa, cyclophosphamide and autologous stem cell transplantation in first-line treatment of metastatic breast cancer. Bone Marrow Transplant. 2008; 41:555-62
14. Lotz JP, Cure H, Janvier $M$, et al: High-dose chemotherapy with haematopoietic stem cell transplantation for metastatic breast cancer patients: final results of the French multicentric randomised CMA/PEGASE 04 protocol. Eur J Cancer. 2005; 41:71-80.

15. Vredenburgh JJ, Coniglio D, Broadwater G, et al: Consolidation with high-dose combination alkylating agents with bone marrow transplantation significantly improves disease-free survival in hormone-insensitive metastatic breast cancer in complete remission compared with intensive standard-dose chemotherapy alone. Biol Blood Marrow Transplant. 2006; 12:195-203.

16. Vredenburgh JJ, Madan B, Coniglio D, et al: A randomized phase III comparative trial of immediate consolidation with high-dose chemotherapy and autologous peripheral blood progenitor cell support compared to observation with delayed consolidation in women with metastatic breast cancer and only bone metastases following intensive induction chemotherapy. Bone Marrow Transplant. 2006; 37:1009-15.

17. Stadtmauer EA, O'Neill A, Goldstein LJ, et al: Conventional-dose chemotherapy compared with high-dose chemotherapy plus autologous hematopoietic stem-cell transplantation for metastatic breast cancer. Philadelphia Bone Marrow Transplant Group. N Engl J Med. 2000; 342:1069-76.

18. Schechter $\mathrm{AL}$, Hung $\mathrm{MC}$, Vaidyanathan $\mathrm{L}$, et al: The neu gene: an erbB-homologous gene distinct from and unlinked to the gene encoding the EGF receptor. Science. 1985; 229:976-8.

19. $\mathrm{Yu}$ D, Hamada J, Zhang $\mathrm{H}$, et al: Mechanisms of c-erbB2/neu oncogene-induced metastasis and repression of metastatic properties by adenovirus 5 E1A gene products. Oncogene. 1992; 7:2263-70.

20. Slamon DJ, Godolphin W, Jones LA, et al: Studies of the HER-2/neu proto-oncogene in human breast and ovarian cancer. Science. 1989; 244:707-12.

21. Slamon DJ, Clark GM, Wong SG, et al: Human breast cancer: correlation of relapse and survival with amplification of the HER-2/neu oncogene. Science. 1987; 235:177-82.

22. Berchuck A, Kamel A, Whitaker R, et al: Overexpression of HER-2/neu is associated with poor survival in advanced epithelial ovarian cancer. Cancer Res. 1990; 50:4087-91.

23. Romond EH, Perez EA, Bryant $\mathrm{J}$, et al: Trastuzumab plus adjuvant chemotherapy for operable HER2-positive breast cancer. N Engl J Med. 2005; 353:1673-84.

24. Perez EA, Romond EH, Suman VJ, et al: Four-year follow-up of trastuzumab plus adjuvant chemotherapy for operable human epidermal growth factor receptor 2-positive breast cancer: joint analysis of data from NCCTG N9831 and NSABP B-31. J Clin Oncol. 2011; 29:3366-73.

25. Piccart-Gebhart MJ, Procter M, Leyland-Jones B, et al: Trastuzumab after adjuvant chemotherapy in HER2-positive breast cancer. N Engl J Med. 2005; 353:1659-72.

26. Smith I, Procter M, Gelber RD, et al: 2-year follow-up of trastuzumab after adjuvant chemotherapy in HER2-positive breast cancer: a randomised controlled trial. Lancet. 2007; 369:29-36.

27. Joensuu H, Kellokumpu-Lehtinen PL, Bono P, et al: Adjuvant docetaxel or vinorelbine with or without trastuzumab for breast cancer. N Engl J Med. 2006; 354:809-20.

28. Joensuu $\mathrm{H}$, Bono $\mathrm{P}$, Kataja $\mathrm{V}$, et al: Fluorouracil, epirubicin, and cyclophosphamide with either docetaxel or vinorelbine, with or without trastuzumab, as adjuvant treatments of breast cancer: final results of the FinHer Trial. J Clin Oncol. 2009; 27:5685-92.

29. Slamon D, Eiermann $\mathrm{W}$, Robert $\mathrm{N}$, et al: Adjuvant trastuzumab in HER2-positive breast cancer. N Engl J Med. 2011; 365:1273-83.

30. Kim YS, Konoplev SN, Montemurro F, et al: HER-2/neu overexpression as a poor prognostic factor for patients with metastatic breast cancer undergoing high-dose chemotherapy with autologous stem cell transplantation. Clin Cancer Res. 2001; 7:4008-12.

31. Rahman Z, Kavanagh J, Champlin R, et al: 12 cycles of paclitaxel immediately following high-dose chemotherapy with autologous stem cell transplantation can induce complete remissions in patients with advanced breast canc ovarian carcinoma. Proc Annu Meet Am Soc Clin Oncol. 1998

32. Greenberg PA, Hortobagyi GN, Smith TL, et al: Long-term follow-up of patients with complete remission following combination chemotherapy for metastatic breast cancer. J Clin Oncol. 1996; 14:2197-205.

33. Ueno NT, Yu D, Hung MC: Chemosensitization of HER-2/neu-overexpressing human breast cancer cells to paclitaxel (Taxol) by adenovirus type 5 E1A. Oncogene. 1997; 15:953-60.

34. Yu D, Liu B, Tan M, et al: Overexpression of c-erbB-2/neu in breast cancer cells confers increased resistance to Taxol via mdr-1-independent mechanisms. Oncogene. 1996; 13:1359-65.

35. Slamon DJ, Leyland-Jones B, Shak S, et al: Use of chemotherapy plus a monoclonal antibody against HER2 for metastatic breast cancer that overexpresses HER2 N Engl J Med. 2001; 344.783-92.

36. Seidman A, Hudis C, Pierri MK, et al: Cardiac dysfunction in the trastuzumab clinical trials experience. J Clin Oncol. 2002; 20:1215-21.

37. Cameron D, Casey M, Oliva C, et al: Lapatinib plus capecitabine in women with HER-2-positive advanced breast cancer: final survival analysis of a phase III randomized trial. Oncologist. 2010; 15:924-34.

38. Blackwell KL, Burstein $\mathrm{HJ}$, Storniolo AM, et al: Randomized study of Lapatinib alone or in combination with trastuzumab in women with ErbB2-positive, trastuzumab-refractory metastatic breast cancer. J Clin Oncol. 2010; 28:1124-30. 
39. Blackwell KL, Burstein HJ, Storniolo AM, et al: Overall survival benefit with lapatinib in combination with trastuzumab for patients with human epidermal growth factor receptor 2-positive metastatic breast cancer: final results from the EGF104900 Study. J Clin Oncol. 2012; 30:2585-92.

40. Baselga J, Cortes J, Kim SB, et al: Pertuzumab plus trastuzumab plus docetaxel for metastatic breast cancer. N Engl J Med. 2012; 366:109-19.

41. Gianni L, Pienkowski T, Im YH, et al: Efficacy and safety of neoadjuvant pertuzumab and trastuzumab in women with locally advanced, inflammatory, or early HER2-positive breast cancer (NeoSphere): a randomised multicentre, open-label, phase 2 trial. Lancet Oncol. 2012; 13:25-32.

42. Verma S, Miles D, Gianni L, et al: Trastuzumab emtansine for HER2-positive advanced breast cancer. N Engl J Med. 2012; 367:1783-91. 